\title{
Predicting cardiorespiratory instability
}

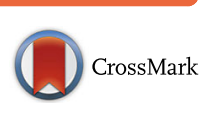

\author{
Michael R. Pinsky ${ }^{1,2^{*}}$, Gilles Clermont ${ }^{1}$ and Marilyn Hravnak ${ }^{3}$
}

\begin{abstract}
This article is one of ten reviews selected from the Annual Update in Intensive Care and Emergency medicine 2016. Other selected articles can be found online at http://www.biomedcentral.com/collections/ annualupdate2016. Further information about the Annual Update in Intensive Care and Emergency Medicine is available from http://www.springer.com/ series/8901.
\end{abstract}

\section{Background}

Identification of patients with overt cardiorespiratory insufficiency or at high risk of impending cardiorespiratory insufficiency is often difficult outside the venue of directly observed patients in highly staffed areas of the hospital, such as the operating room, intensive care unit (ICU) or emergency department. And even in these care locations, identification of cardiorespiratory insufficiency early or predicting its development beforehand is often challenging. The clinical literature has historically prized early recognition of cardiorespiratory insufficiency and its prompt correction as being valuable at minimizing patient morbidity and mortality while simultaneously reducing healthcare costs. Recent data support the statement that integrated monitoring systems that create derived fused parameters of stability or instability using machine learning algorithms, accurately identify cardiorespiratory insufficiency and can predict their occurrence. In this overview, we describe integrated monitoring systems based on established machine learning analysis using various established tools, including artificial neural networks, k-nearest neighbor, support vector machine, random forest classifier and others on routinely acquired non-invasive and invasive hemodynamic measures to identify cardiorespiratory insufficiency and display them in real-time with a high degree of precision.

\footnotetext{
*Correspondence: pinsky@pitt.edu

'Department of Critical Care Medicine, University of Pittsburgh, 15261 Pittsburgh, PA, USA

2Department of Anesthesiology, University of California, San Diego, La Jolla, CA, USA

Full list of author information is available at the end of the article
}

The implications of these approaches for all healthcare monitoring across the spectrum of in-patient to chronic care is clear, even though the need may appear more pressing for the acute care setting to those of us whose daily life is centered there. The underlying assumption of these approaches is that measured changes in easily acquired physiologic variables reflect complex patientspecific interactions amongst multiple regulatory autonomic, hormonal and metabolic systems. Accordingly, simple algorithm approaches to such interactions, like the use of the existing severity scoring systems or computer-based treatment protocols, are unlikely to improve outcomes other than by standardizing therapies. Potentially, by using functional hemodynamic monitoring principles, previously described and validated, we can predict with a high degree of accuracy volume responsiveness and central arterial tone in all patients. But one needs to identify who is unstable or going to be unstable before applying these functional hemodynamic monitoring approaches.

\section{Integrated monitoring systems improve diagnosis of cardiorespiratory insufficiency and treatment effectiveness}

Current resuscitation decisions are typically made in response to a falling blood pressure (BP), persistently high heart rate $(\mathrm{HR})$ or arterial desaturation [1]. Individual vital signs (BP, HR, respiratory rate, pulse oximeter oxygen saturation $\left[\mathrm{SpO}_{2}\right]$, and end-tidal $\mathrm{CO}_{2}$ ) are usually assessed as mean values and interpreted independently. These point estimates may not reach an actionable level until the patient has already progressed to late (or decompensated) cardiorespiratory insufficiency. Alternatively, an integrated monitoring system can use fused data, collected and synthesized to identify physiologic patterns, which are predictive of instability in real-time and before overt clinical deterioration [2]. To see whether such an integrated monitoring system could identify overt cardiorespiratory insufficiency earlier in its course and reduce overall patient instability, we used a Food and Drug Administration (FDA)-approved Visensia ${ }^{\mathrm{TM}}$ monitor (OBS Medical, Carmel, IN) that amalgamates non-invasive vital signs (BP, $\mathrm{HR}$, respiratory rate, $\mathrm{SpO}_{2}$ ) and derives a calculated 
index score (vital signs index [VSI]) between zero and ten using an artificial neuronet approach [3]. Using our 600 step-down unit (SDU) patient cohort as a calibration set, we recalibrated the VSI algorithm to fit our cohort, wherein VSI values of $>3.2$ correlated significantly with independently estimated instability based on clinical assessment $(r=0.815)$ [4]. An example of one patient who progressively deteriorated over a $6 \mathrm{~h}$ period is shown in Fig. 1. Note that deterioration is not steady but phasic (blue arrows) with periods of failed recovery ending in collapse (black arrow). Furthermore, when the VSI alert was coupled with an effector arm of direct immediate nursing bedside activation and protocolized treatment, overall instability decreased by $150 \%$ and the progression from mild to severe instability was reduced by $300 \%$ $[5,6]$. Importantly, the VSI alert occurred before clinically-apparent instability in $80 \%$ of cases, with an advance time of $9.4 \pm 9.2 \mathrm{~min}$. Thus, such bedside-displayed VSI data can often detect the onset of cardiorespiratory insufficiency before overt symptoms are present and when coupled to appropriate immediate treatment plans markedly reduces patient instability.

Demographic and clinical characteristics are useful in predicting mortality for groups of patients using static snapshot models such as APACHE III [7] or IV [8], and also help to predict mortality when added to intermittent vital sign amalgamation. Smith et al. [9] determined that adding age to a single-parameter instability-concern model $(\mathrm{RR}<5$ or $>36 / \mathrm{min}$, HR $<40$ or $>140 / \mathrm{min}$, systolic $\mathrm{BP}<90 \mathrm{mmHg}$, sudden fall in level of consciousness) or the intermittently determined Modified Early Warning System (MEWS) improved mortality prediction. Patients $\geq 80$ years of age with a RR of 24-25 per minute had 4 times the mortality of patients $40-64$ years of age, and those $\geq 80$ years of age with a systolic BP of $90-94 \mathrm{mmHg}$ had 10 times the mortality of those aged 40-64 years of age. Higher age also increased mortality prediction as MEWS score increased. We subsequently validated this improved predictive index in our SDU cohort, wherein adding low frequency data (demographics) markedly improved the predictive value of the VSI alerts in SDU patients [10].

\section{Advanced signal processing improves predictive value of HR for identifying impending instability}

Batchinsky et al. showed that advanced signal processing $\mathrm{R}-\mathrm{R}$ intervals could be used to predict trauma survivorship [11]. They then showed clear differences in HR complexity in 31 pre-hospital trauma patients during their helicopter transport to a level 1 trauma center who survived (20 survived) or died (11 died) after admission. Although mean HR was not different between groups (117 \pm 9 vs. $100 \pm 4 / \mathrm{min}$, non-survivors vs. survivors), their HR variability, quantified by the instantaneous $R-R$

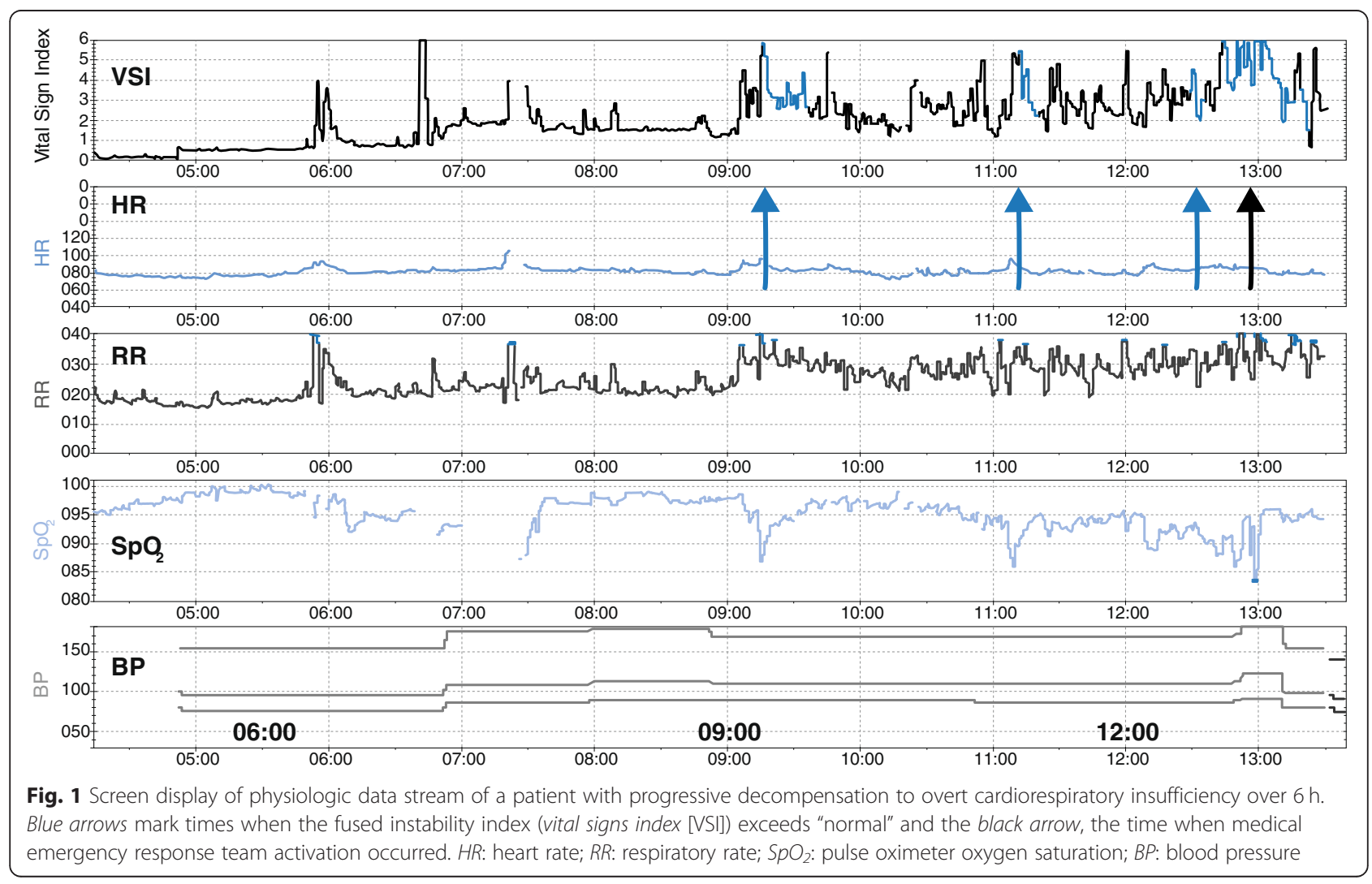


interval changes on a beat-to-beat basis were clearly different. They quantified HR variability by assessing approximate entropy (ApEn), sample entropy (SampEn) and similarity of distributions. Traditionally, heart rate variability, estimated, as the standard deviation of the R-R interval, requires at least 800 beats to derive robust values. However, these authors showed that by using these derived parameters of variability, SampEn not only displayed clear separation of values between survivors and non-survivors, but the strength of the discrimination persisted even when the datasets were reduced from 800 to $100 \mathrm{R}-\mathrm{R}$ intervals [11]. Furthermore, these electrocardiographic (EKG)-derived signal differences were also associated with the need for life-saving interventions in these same trauma patients [12]. Finally, they verified the above findings in a mixed cohort of prehospital trauma patients [13]. Thus, readily available vital sign data can be used to derive clinically-relevant prediction parameters with precision and a markedly reduced lead time.

\section{Advanced signal processing of physiologic variable time series identifies those SDU patients who will become unstable from those who will not}

Using the above SDU patient data series [5, 6], we analyzed $H R$ variability parameters similar to those described by Batchinsky et al. [11-13]. We created a HR variability index based on HR autocorrelation, standard deviation, high frequency power of HR frequency spectrum and ApEn. The resulting fused parameter was significantly different for the 80 patients in the 307 patient cohort who experienced at least one cardiorespiratory insufficiency episode. Importantly, when displayed as $5 \mathrm{~min}$ epochs moving backward from the instability event or discharge in the two groups, HR variability discriminated between the two groups $>48 \mathrm{~h}$ before these events (Fig. 2) [14]. Thus, advanced signal processing of clinical data can identify instability before it becomes clinically apparent, often with many hours of lead time.

\section{Advanced monitoring-derived comprehensive libraries}

It is not enough to use existing data streams to predict instability. One must also create physiological libraries of complex and dynamic states, such as hemorrhage, sepsis, pump failure, or evolving acute lung injury (ALI). Normal physiological reflexes aggressively support blood flow to the heart and brain and thus may well obfuscate bedside assessment. We used highly instrumented animal models to define high fidelity physiologic patterns of individual animal response to disease. We studied these patterns of response in compensated trauma/hemorrhagic shock, both during the progression to cardiovascular collapse and its response to resuscitation therapies. As with the above vital sign analysis, we note not only the absolute values of measured hemodynamic variables ascertained from non-invasive and minimally invasive biosensors, but also their dynamic response to prescribed physiological challenges. Compensation, exhaustion and response to therapy reflect the three primary processes studied.

The experimental hemorrhage protocol is designed to simulate a dynamically changing clinical situation by modifying a Wigger's model using several discrete bleeding episodes based on the animal's physiologic response. Lightly anesthetized swine followed an arterial pressuredriven experimental hemorrhage protocol to a mean arterial pressure (MAP) of $30 \mathrm{mmHg}$, held there for a maximum of $90 \mathrm{~min}$ then resuscitated. The porcine trauma/hemorrhagic shock model plays into the unique nature of each test animal by having the level of bleed defined by the subsequent MAP and not by the amount of blood shed. This allowing us to examine the specific compensating mechanisms, unique measures of decompensation and tissue viability and response to therapy [15]. High fidelity $(256 \mathrm{~Hz})$ hemodynamic waveform collection and low frequency endocrine, metabolic and immunologic parameters can also be recorded throughout the experiment. Instrumentation with additional biosensors to assess tissue $\mathrm{O}_{2}$ saturation $\left(\mathrm{StO}_{2}\right)$, tissue $\mathrm{CO}_{2}$ and $\mathrm{pH}$, capillary blood flow and mucosal $\mathrm{NADH}_{2}$ levels were also performed as well as dynamic stress tests described below. The partial list of 'non-traditional' biosensors we have used in this model and that can be used clinically going forward is given in Table 1.

The cause of cardiovascular collapse from compensated trauma/hemorrhagic shock appears to be related to failure of compensatory response mechanisms rooted in autonomic balance. Trauma/hemorrhagic shock acts as a trigger for a cascade of post-traumatic events involving hemodynamic, neuro-endocrine and inflammatory systems interactions, among others. Such varied multifactorial interactions lend themselves to complexity modeling because analyses performed to identify the onset of cardiovascular collapse reflect variable interactions rather than single parameter changes. Thus, the intrinsic variability of response among subjects that makes linear analysis of trauma/hemorrhagic shock difficult is actually a desired quality to build a predictive complexity model. The normal interaction between measured variables will be altered by responses to pathological insults. For example, failure of sympathetic drive and related endocrine response to trauma/hemorrhagic shock account for refractoriness to conventional resuscitation [16-21]. Failure of sympathetic/endocrine coupling effectors (e.g., epinephrine) and vascular endothelial-smooth muscle coupling may explain cardiovascular refractoriness and cardiovascular collapse in trauma/hemorrhagic shock [22]. Cellular energetic failure through impaired mitochondrial oxidative phosphorylation may further explain the vasodilatation seen in late stages of hemorrhagic 


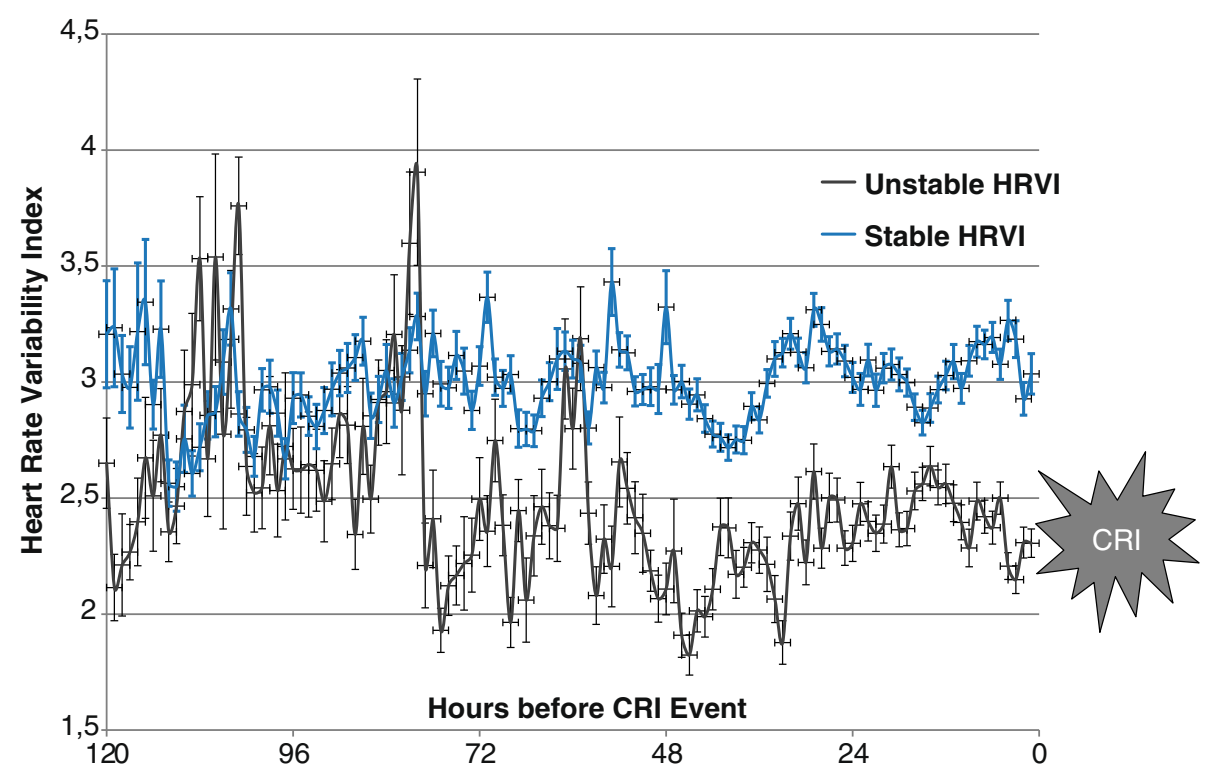

Fig. 2 Heart rate variability index (HRVI) of step-down unit patients who were never unstable (blue) and those who would become unstable (gray) at time zero. Times are displayed preceding instability events for the patients wo would become unstable and a similar time frame but at random to hospital stay for a matched cohort of stable step-down unit patients. CRl: cardiorespiratory instability

shock similar to that reported in septic shock [23, 24]. Elevated $\mathrm{NADH}_{2}$ levels mirror hypotension but often persist for several minutes during resuscitation despite restoration of MAP [25].

\section{Extending biosensor utility using functional hemodynamic monitoring for prediction}

Fully half of all hemodynamically unstable ICU patients are not volume-responsive [26]. Estimates of preload (e.g., right $[\mathrm{RV}]$ or left $[\mathrm{LV}]$ ventricular volumes, intrathoracic blood volume or ventricular filling pressures) do not predict volume-responsiveness. Functional hemodynamic monitoring overcomes this limitation of traditional hemodynamic monitoring [27, 28]. In this case, functional hemodynamic monitoring uses a small volume loading challenge to perturb the cardiovascular autoregulatory function. Examples of preload challenges validated in multiple clinical trials include small rapid bolus volume infusions (i.e., fluid challenge), positivepressure breathing [29] and passive leg-raising (PLR) to $30^{\circ}$ [30]. If LV stroke volume increases transiently with these maneuvers, then cardiac output will also increase with subsequent fluid infusion. The degree of increase is quantified as the ratio of the maximal change in pulse pressure or stroke volume over $4-5$ breaths or with PLR to the mean pulse pressure or stroke volume, referred to as pulse pressure variation (PPV) or stroke volume variation (SVV), respectively. The disadvantages of a traditional fluid challenge are that it takes time, often is given too slowly, thus masking volume responders,

Table 1 FDA-approved non-invasive non-traditional biosensors available and previously used by us

\begin{tabular}{lll}
\hline Sensor name & Parameters measured & Manufacturer \\
\hline Trendcare Multiparameter & Tissue $\mathrm{PCO}_{2}, \mathrm{PO}_{2}, \mathrm{pH}$ & Diametrics Medical \\
CritiView CRV3 & Mitochondrial function ( $\mathrm{NADH}_{2}$ fluorescence), microcirculatory & CritiSence Inc. \\
InSpectra & blood flow, volume and oxygenation & Hutchinson Industry \\
$\mathrm{CV}$ InSight & Tissue $\mathrm{O}_{2}$ saturation & iNTELOMED \\
Microscan & Vascular tone & Microvision Medical \\
Cytoscan & Microcirculatory flow & Cytometrics \\
Clearsight finger plethysmograph & Microcirculatory flow & Edwards Lifesciences \\
CNAP finger plethysmograph & Blood pressure and cardiac output & cnsystems \\
NICOM & Blood pressure and cardiac output & Cheetah Medical \\
Navigator-1 & Cardiac output, stroke volume variation, thoracic fluid & Applied Physiology \\
\hline
\end{tabular}


and is irreversible. Functional hemodynamic monitoring techniques give reliable predictions of preload response immediately and do not require fluid infusions to make this prediction. Both positive-pressure ventilation by physically decreasing venous return with inspiration and leg-raising by transiently increasing venous return fulfill these criteria [29, 30]. PPV and SVV can be easily monitored using several FDA-approved minimally invasive monitoring devices. Thus, in patients receiving positive-pressure breathing, simple inspection of the arterial PPV will continuously define volume responsiveness. The magnitude of PPV and SVV during ventilation will also be a function of the size of the tidal breath [31], thus this approach is only useful during controlled mechanical ventilation at a fixed tidal volume, which is not the case in spontaneously breathing patients. Furthermore, PPV and SVV do not reflect volume responsiveness in patients with atrial fibrillation where R-R intervals vary widely. However, a PLR maneuver with leg elevation to $30^{\circ}$ displays the same predictive information in all patients [30]. We and others have extensively documented that a PPV $>13 \%$ or a SVV $>10 \%$ at a tidal volume of $7 \mathrm{ml} / \mathrm{kg}$ or a maximal increase in mean cardiac output $>10 \%$ during a PLR maneuver are predictive of preload responsiveness (>90\% sensitivity and specificity) [32]. Several minimally-invasive devices report cardiac output, PPV and SVV during positive-pressure breathing or change in cardiac output with PLR using arterial waveform analysis (e.g., PiCCO plus ${ }^{\text {Tm }}$ [Pulsion Medical Systems], LiDCO plus $^{\mathrm{ma}}$ and LiDCO rapid $^{\mathrm{m} x}$ [LiDCO Group Plc] and Flo$\operatorname{Trac}^{\text {TM }}$ [Edwards Lifesciences]). We have previously defined the operating characteristics and reliability of all these devices [33, 34]. Finally, the PPV/SVV ratio reflects central arterial elastance and can be used to monitor changes in vasomotor tone [12].

Importantly, FDA-approved non-invasive surrogate estimates of arterial pulse pressure and stroke volume exist, including pulse oximetry signal, bioreactance (NICOM, Cheetah) and transthoracic ultrasound (USCom) techniques. Pulse oximetry density profiles derived from the unprocessed pulse oximetry plethysmographic waveform amplitude (Nonin, Nelcor and Massimo), and pressuresensitive optical sensors (BMEYE, Edwards Lifesciences) can be featurized to estimate pulse pressure, stroke volume and changing vasomotor tone [35]. The BMEYE pressure-sensitive, high fidelity, rapid-response optic sensor has the ability to track the arterial pressure profile to measure instantaneous cardiac output [36] and, along with the pulse oximetry plethysmographic profile, reflect two real-time waveform signals that we can use to extract predictive features of the cardiovascular system. Importantly, these non-invasive waveform data can be analyzed independent of their mean values and expand the utility of these analyses and predictive modeling beyond invasive monitoring to less invasive monitoring environments, markedly increasing generalizability of this featurized approach.

\section{Non-invasive measures of oxygen sufficiency}

An unanswered question in shock resuscitation is the relationship between tissue perfusion and wellness. Neither MAP, cardiac output or mixed venous oxygen saturation $\left(\mathrm{SvO}_{2}\right)$ define tissue oxygenation. Near infra-red spectroscopy (NIRS) permits continuous, non-invasive measurement of $\mathrm{StO}_{2}$. Although $\mathrm{StO}_{2}$ values do not decrease until tissue perfusion is very low, $\mathrm{StO}_{2}$ becomes more sensitive and specific when monitoring its change in response to a vascular occlusion test (VOT) (Fig. 3). If the $\mathrm{StO}_{2}$ probe is placed on the thenar eminence and a downstream arm blood pressure cuff is inflated to a pressure higher than systolic arterial pressure and held there, total ischemia occurs. The occlusion is sustained until $\mathrm{StO}_{2}$ decreases to $<40 \%$ and then the cuff rapidly deflated. The $\mathrm{StO}_{2}$ down slope is dependent on local metabolic rate and blood flow distribution. The $\mathrm{StO}_{2}$ recovery rate assesses cardiovascular reserve, as we validated in trauma and septic patients compared to normal volunteers [37].

\section{Predicting the need for life-saving interventions in stat medevac air transport}

We assessed the predictive value of the $\mathrm{VOT} \mathrm{StO}_{2}$ and spot lactate levels in trauma patients during emergency air transport from an accident site. All patients were monitored using 3-lead EKG, non-invasive $\mathrm{BP}, \mathrm{HR}, \mathrm{SpO}_{2}$, and when intubated, end-tidal $\mathrm{CO}_{2}$ capnography. These single vital signs are not sensitive at identifying shock until advanced [38]. Protocol-based algorithms typically rely on individual vital signs or clinical parameters (e.g., cyanosis,

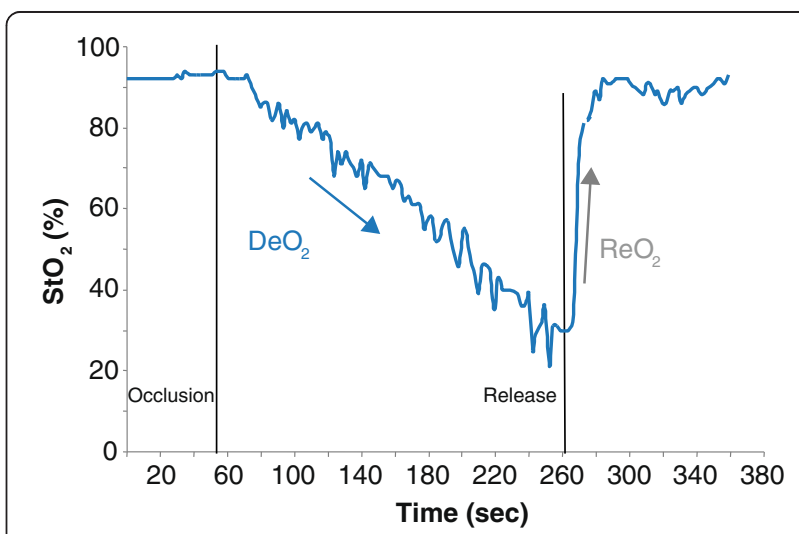

Fig. 3 Graphic display of the thenar tissue oxygen saturation $\left(\mathrm{StO}_{2}\right)$ at baseline and in response to a total vascular occlusion to a minimal $\mathrm{StO}_{2}$ then release, referred to as a vascular occlusion test. Deoxygenation post-occlusion $\left(\mathrm{DeO}_{2}\right)$ and reoxygenation upon release $\left(\mathrm{ReO}_{2}\right)$ 
altered mental status) to identify the need for life-saving interventions [39, 40] and subjective measures (mental status changes) are difficult to standardize [41]. We hypothesized that in-flight measures of VOT $\mathrm{StO}_{2}$ and lactate would identify shock trauma subjects in need of life-saving interventions [42, 43]. We studied 400 transported trauma patients with lactate sampling and 194 patients also with $\mathrm{VOT} \mathrm{StO}_{2}$. Patients with pre-hospital lactate levels $>4 \mathrm{mmol} / \mathrm{l}$ had greater need for emergent operation, intubation, and vasopressor $(p=0.02)$. This association persisted after adjustment for age, Glasgow Coma Scale (GCS) score and initial vital signs. The VOT $\mathrm{StO}_{2}$ deoxygenation slopes were predictive of the need for life-saving interventions $(p=0.007)$, while a delayed reoxygenation slope was predictive of mortality $(p=0.006)$ [44]. These data collectively document that the measurement of readily available physiological variables when coupled to functional hemodynamic monitoring principles (PLR and VOT) can predict clinically relevant physiological states and the subsequent need for life-saving interventions.

\section{Using machine learning principles to define health and disease}

One never truly sees hypovolemia, sepsis, heart failure or ALI in the critically ill patients under our care, one sees the phenotypic physiological response of the host to these pathological processes. Thus, a fundamental aspect of both traditional monitoring and any novel approach is to identify normal biological variability and separate it out from adaptive/reflexive responses and pathological sequelae of these primary processes. For identification and predictive purposes this is very useful because most pathological process presenting as circulatory shock and respiratory insufficiency evolve over time. For example, hypovolemia in the setting of active intravascular volume loss starts with no measurable changes because the volume loss is so small. However, with progressive volume loss by any mechanism (hemorrhage, 3rd space loss, diarrhea), adaptive processes and hemodynamic phenotypic signatures evolve which may not be easily identified early on using primary mean hemodynamic values. However, derived parameters, based on validated machine learning approaches, such as the artificial neuronet of interacting variables or SampEn of time series single source data, can markedly improve the early identification of critical illness. Thus, we hypothesize that by advanced analysis of existing biological data series, one can detect adaptive and maladaptive processes earlier than we presently do such that definitive therapy can be started to reverse these processes before they become severe, induce remote organ injury or become irreversible. For example, an acute asthma attack can often be easily reversible with simple inhalational bronchodilators, whereas if the same process is left untreated until severe status asthmaticus, much more aggressive therapies need to be given to reverse the same process. And this disease and those required therapies (e.g., steroids) markedly increase morbidity and mortality.

Thus, the process of creating accurate sensitive and specific alerts and decision support systems is both iterative and based on creating libraries of 'normal' and 'notnormal' physiological interactions or 'behaviors', and to have a deeper understanding of the fuzziness of the boundary of normality for each of these behaviors. For example, one could use the previously described baseline porcine data prior to trauma/hemorrhagic shock to 'train' the model as to normal biological variability. We will then use the bleeding time, changes in endotoxin infusion, burn or smoke inhalation as time-dependent pathological stressors to calibrate the 'not-normal' states, as described below. We then use these relatively pure pathological insults to define process-specific signatures of disease to identify both the pathological process and its severity. Inherent in this analysis is that if therapy reverses these pathological processes, the derived measure of disease also decreases.

Three major barriers arise when iterating clinical data based on animal experimental data. First, our patient cohorts are often not previously healthy and then subjected to a defined relatively pure insult. They arrive in varying states of illness, preexisting co-morbidities and ongoing therapies. Using a young trauma cohort for initial model development may minimize this effect. Second, human data are typically not as rich in terms of frequency and number of variables collected given field conditions and other pragmatic reasons. Patients get disconnected from monitoring devices for various reasons (e.g., X-rays, turning), EKG electrodes and pulse oximeter probes fall off and primary signals can be inaccurate (clotted catheter). Thus, an initial data processing aspect of any model building needs to review these data streams and identify gaps in data flow and artifacts. Finally, one cannot truly define 'normal' in our critically ill patients, only normal behavior. For example, an animal in hemorrhagic shock may appear to be normal based on measured variables and derived parameters if they are also getting vasopressor therapy. Thus, the best we can do across all pathophysiological domains is to report not-normal and stability, both of which must be interpreted within the context of therapy.

Within these constraints, one must first determine the minimal data set (independent monitored signal, sampling frequency and lead time) required to identify not-normal with an acceptable level of false alerts and long enough lead time to overt disease expression as to be clinically relevant. We refer to this approach as "hemodynamic monitoring parsimony". Intuitively, one expects tradeoffs 
between parsimony, lead time and accuracy. Initially, a 15 min advanced warning may be the minimal lead time for cardiorespiratory instability to be clinically relevant. Once an alert of not-normal is made, one may sequentially insert additional measures to determine their ability to improve sensitivity and specificity of these alerts in defining specific disease processes so as to guide therapy. The concept of monitoring parsimony extends beyond hemodynamic monitoring. As the ability to merge hemodynamic data with other clinically relevant data streams, it is expected that a parsimonious set of clinical features useful to cardiorespiratory instability detection and prediction will include non-hemodynamic data as well.

How do we put all of this together? At the mathematical level there are two main problems. The first is how to predict the occurrence of events in data rich scenarios, such as in our porcine data, and the second is how to do the same in humans, which usually will involve only a few biomarkers. In addition, why do we need animal models to predict human behavior? Cannot this analysis be done completely on the human data using only a few biomarkers such as $\mathrm{BP}, \mathrm{HR}$, respiratory, $\mathrm{SpO}_{2}$ and minimally invasive measures? Our preliminary analysis of the porcine dataset, which involves many biomarkers, and VSI human data involving the four physiological variables mentioned above [4-6] suggest that our insight can be improved tremendously by using the animal data. It is possible that the variables we use now from the animal trauma/hemorrhagic shock model are not the best for human instability prediction. For example, the grouping and its variation over time is not apparent in the small human dataset we collected of trauma SDU patients [6]. The animal models that are very close to human disease will allow us to gain a much better understanding of the dynamic features and which of these are the important players at different stages of stress, compensation, resuscitation, recovery and death. These animal analyses may also suggest which variables can be omitted in certain cases, and what omission implies about disease level and adaptation, etc. We hypothesize that data-driven prediction modeling approaches will enable healthcare professionals both at the bedside and in remote settings to predict those patients most likely to develop future instability. We also hypothesize that dynamic systems modeling will further improve prediction, including the provision of various signatures for instability subtype. This is and will continue to be an amazing and informative journey.

\section{Conclusions}

Machine learning principles when coupled with a knowledge of human physiology, pathophysiology can create highly informative displays and alerts. Such informaiot can be in the form of anomaly detection, defining the switch from health to disease, may also be disease specific and can track responses to treatment and time. The goals of these efforts is to glean knowledge from data to improve patient care across the spectrum of patient monitoring environments. The future will need to focus on creating a common dictionary for healthcare, common data elements and methods of structuring the data and ways of sharing large data sets that retain patient confidentiality without sacrificing detail.

\section{Competing interests}

Michael R. Pinsky, MD is the inventor of a University of Pittsburgh owned US Patent: Use of complexity modelling to predict cardiorespiratory insufficiency. Gilles Clermont, MD none declared.

Marilyn Hravnak, BSN, PhD none declared.

\section{Authors' contributions}

All authors read and approved the final manuscript.

\section{Declarations}

This work and its publication was supported in part by NIH grant NR013912.

\section{Author details}

${ }^{1}$ Department of Critical Care Medicine, University of Pittsburgh, 15261 Pittsburgh, PA, USA. '2Department of Anesthesiology, University of California, San Diego, La Jolla, CA, USA. ${ }^{3}$ Department of Tertiary Care Nursing, University of Pittsburgh, Pittsburgh, PA 15261, USA.

Published online: 16 March 2016

\section{References}

1. Goodrich C. Endpoints of resuscitation: what should we be monitoring? AACN Adv Crit Care. 2006;17:306-16.

2. Hravnak M, DeVita M, Edwards L, Clontz A, Valenta C, Pinsky MR. Cardiorespiratory instability before and after implementing an integrated monitoring system. Am J Respir Crit Care Med. 2008;177:A842 (abst).

3. Tarassenko L, Hann A, Young D. Integrated monitoring and analysis for early warning of patient deterioration. Br J Anaesth. 2006;97:64-8.

4. Hravnak M, Edwards L, Clontz A, Valenta C, DeVita M, Pinsky MR. Defining the incidence of cardio-respiratory instability in step-down unit patients using an electronic integrated monitoring system. Arch Intern Med. 2008; 168:1300-8

5. Hravnak M, Edwards L, Foster-Heasley M, et al. Electronic integrated monitoring of medical emergency team calls to a step down unit. Circulation. 2007;116(II):939.

6. Hravnak M, DeVita MA, Clontz A, Edwards L, Valenta C, Pinsky MR. Cardiorespiratory Instability Before and After Implementing an Integrated Monitoring System. Crit Care Med. 2011;39:65-72.

7. Knaus WA, Wagner DP, Draper EA, et al. The APACHE III prognostic system. Risk prediction of hospital mortality for critically ill hospitalized adults. Chest. 1991;100:1619-36.

8. Zimmerman JE, Kramer AA, McNair DS, Malila FM. Acute Physiology and Chronic Health Evaluation (APACHE) IVL hospital mortality assessment for today's critically ill patients. Crit Care Med. 2006;34:1297-310.

9. Smith GB, Prytherch DR, Schmidt PE, et al. Should age be included as a component of track and trigger systems used to identify sick adult patients? Resuscitation. 2008;78:109-15.

10. Yousef K, Pinsky MR, DeVita MA, Sereika S, Hravnak M. Demographic and clinical predictors of cardiorespiratory instability in a step-down unit: pilot study. Am J Crit Care. 2012;21:344-50.

11. Batchinsky Al, Salina J, Kuusela T, Necsoiu C, Jones J, Cancio LC. Rapid prediction of trauma patients survival by analysis of heart rate complexity: impact of reducing data set size. Shock. 2009;32:565-71.

12. Cancio LC, Batchinsky Al, Salinas J, et al. Heart-rate complexity for prediction of prehospital lifesaving interventions in trauma patients. J Trauma. 2008;65:813-9. 
13. Batchinsky Al, Salinas J, Jones JA, Necsoiu C, Cancio LC. Predicting the need to perform life-saving interventions in trauma patients using new vital signs and artificial neural networks. Lecture Notes in Computer Science. Berlin: Springer; 2009

14. Ogundele O, Clermont G, Sileanu F, Pinsky MR. Use of derived physiologic variable to predict individual patients' probability of hemodynamic instability. Am J Respir Crit Care Med. 2013;187:A5067 (abst).

15. Zenker S, Polanco PM, Kim HK, et al. Threshold area over the curve of spectrometric tissue oxygen saturation as an indicator of volume resuscitability in an acute porcine model of hemorrhagic shock. J Trauma. 2007;63:573-80

16. Gannon TA, Britt RC, Weireter LJ, Cole FJ, Collins JN, Britt LD. Adrenal insufficiency in the critically III trauma population. Am Surg. 2006;72:373-6.

17. Porter MH, Cutchins A, Fine JB, Bai Y, DiGirolamo M. Effects of TNF-alpha on glucose metabolism and lipolysis in adipose tissue and isolated fat-cell preparations. J Lab Clin Med. 2002;139:140-6.

18. Helling TS. The liver and hemorrhagic shock. J Am Coll Surg. 2005;201:774-83.

19. Evans DA, Jacobs DO, Wilmore DW. Tumor necrosis factor enhances glucose uptake by peripheral tissues. Am J Physiol. 1989;257:R1 182-9.

20. Chang CG, Van Way 3rd CW, Dhar A, Helling Jr T, Hahn Y. The use of insulin and glucose during resuscitation from hemorrhagic shock increases hepatic ATP. J Surg Res. 2000;92:171-6.

21. Carey LC, Curtin R, Sapira JD. Influence of hemorrhage on adrenal secretion, blood glucose and serum insulin in the awake pig. Ann Surg. 1976;183:185-92.

22. Landry DW, Oliver JA. The pathogenesis of vasodilatory shock. N Engl J Med. 2001;345:588-95.

23. Singer M. Mitochondrial function in sepsis: acute phase versus multiple organ failure. Crit Care Med. 2007;35:441-8.

24. Leverve XM. Mitochondrial function and substrate availability. Crit Care Med. 2007;35:S454-60.

25. Clavijo-Alvarez JA, Sims CA, Soller B, Pinsky MR, Puyana JC. Monitoring skeletal muscle and subcutaneous tissue acid-base status and oxygenation during hemorrhagic shock and resuscitation. Shock. 2005;24:270-5.

26. Michard F, Teboul JL. Predicting fluid responsiveness in ICU patients: A critical analysis of the evidence. Chest. 2002;212:2000-8.

27. Pinsky MR. Hemodynamic evaluation and monitoring in the ICU. Chest. 2007;123:2020-9.

28. Pinsky MR. Functional hemodynamic monitoring: use of derived variable to diagnose and manage the critically ill. Acta Anaesthesiol Scand. 2009:53 suppl 119:9-11.

29. Michard F, Chemla D, Richard C, et al. Clinical use of respiratory changes in arterial pulse pressure to monitor the hemodynamic effects of PEEP. Am J Respir Crit Care Med. 1999;159:935-9.

30. Monnet X, Rienzo M, Osman D, et al. Response to leg raising predicts fluid responsiveness in critically ill. Crit Care Med. 2006;34:1402-7.

31. Kim HK, Pinsky MR. Effect of tidal volume, sampling duration and cardiac contractility on pulse pressure and stroke volume variation during positivepressure ventilation. Crit Care Med. 2008;36:2858-62.

32. Pinsky MR. Functional hemodynamic monitoring: A personal perspective. In: Vincent $J \mathrm{~L}$, editor. Yearbook of Emergency and Intensive Care Medicine. Berlin: Springer; 2009. p. 306-10.

33. Marquez J, McCurry K, Severyn DA, Pinsky MR. Ability of pulse power, esophageal Doppler and arterial pressure to estimate rapid changes in stroke volume in humans. Crit Care Med. 2008;36:3001-7.

34. Hadian M, Kim HK, Severyn D, Pinsky MR. Cross-comparison of continuous cardiac output trending accuracy of LiDCO, PiCCO, FloTrac and pulmonary artery catheters. Crit Care. 2010;14:R212.

35. Cannesson M, Besnard C, Durand PG, Bohé J, Jacques D. Relation between respiratory variations in pulse oximetry plethysmographic waveform amplitude and arterial pulse pressure in ventilated patients. Crit Care. 2005;9:R562-8.

36. Benomar B, Quattara A, Estagnasie P, Brusset A, Squara P. Fluid responsiveness predicted by non-invasive bioreactance-based passive leg raise test. Intensive Care Med. 2010;36:1875-81.

37. Gomez H, Torres A, Zenker S, et al. Use of non-invasive NIRS during vascular occlusion test to assess dynamic tissue $\mathrm{O} 2$ saturation response. Intensive Care Med. 2008;34:1600-7.

38. Wo CCJ, Shoemaker WC, Appel PL, et al. Unreliability of blood pressure and heart rate to evaluate cardiac output in emergency resuscitation and critical illness. Crit Care Med. 1993;21:218-23.
39. Holcomb JB, Niles SE, Miller CC, Hinds D, Duke JH, Moore FA. Prehospital physiologic data and lifesaving interventions in trauma patients. Mil Med. 2005; 170:7-13.

40. Holcomb JB, Salinas J, McManus JJ, Miller CC, Cooke WH, Convertino VA. Manual vital signs reliably predict need for life-saving interventions in trauma patients. J Trauma. 2005;59:821-9.

41. Porter JM, Ivatury RR. In search of the optimal end points of resuscitation in trauma patients: a review. J Trauma. 1998;44:908-14.

42. Guyette FX, Suffoletto BP, Castillio JL, Puyana JC. Identification of occult shock using out-of-hospital lactate. Ann Emerg Med. 2009;54:S142 (abst)

43. Castillio $J L$, Guyette FX, Suffoletto BP, Peitzman AB, Puyana JC. The role of prehospital lactate as a predictor of outcomes in trauma patients. J Trauma. 2009;63:S138 (abst).

44. Guyette F, Gomez H, Suffoletto B, et al. Prehospital dynamic tissue O2 saturation response predicts in-hospital mortality in trauma patients. Crit Care Med. 2009;37(12 Suppl):A28. 